\title{
Distinct Roles for the Two Rho GDP/GTP Exchange Factor Domains of Kalirin in Regulation of Neurite Growth and Neuronal Morphology
}

\author{
Peter Penzes, ${ }^{1}$ Richard C. Johnson, ${ }^{1}$ Vikram Kambampati, ${ }^{1}$ Richard E. Mains, ${ }^{2}$ and Betty A. Eipper ${ }^{2}$ \\ ${ }^{1}$ Department of Neuroscience, Johns Hopkins University School of Medicine, Baltimore, Maryland 21205, and \\ 2Department of Neuroscience, University of Connecticut Health Center, Farmington, Connecticut 06030
}

The actin cytoskeleton, essential for neuronal development, is regulated in part by small GTP binding proteins of the Rho subfamily. Kalirin-9, with two Rho subfamily-specific GDP/GTP exchange factor (GEF) domains, localizes to neurites and growth cones of primary cortical neurons. Kalirin-9 overexpression in cultured cortical neurons induces longer neurites and altered neuronal morphology. Expression of the first GEF domain alone results in drastically shortened axons and excessive growth cones, mediated by Rac1. Expression of the second

The actin cytoskeleton plays an essential role in morphological changes during neuronal development and plasticity. Rho GTPases are key regulators of the actin cytoskeleton (Hall, 1998), and there is ample evidence involving Rho GTPases in growth of neurites and axon guidance (Luo et al., 1994; Threadgill et al., 1997; Li et al., 2000). The immediate activators of Rho-like GTPases are GDP/GTP exchange factors (GEFs). All GEFs for Rho subfamily members contain a catalytic Dbl-homology (DH) domain, followed immediately by a pleckstrin homology $(\mathrm{PH})$ domain (Whitehead et al., 1997). Neuronal GEFs include proteins with two DHPH domains, such as Kalirin (Alam et al., 1997; Johnson et al., 2000; Penzes et al., 2000), Trio (Debant et al., 1996), Caenorhabditis elegans UNC-73 (Steven et al., 1998), and Drosophila Trio (Awasaki et al., 2000; Bateman et al., 2000; Liebl et al., 2000; Newsome et al., 2000) (see Fig. 1a).

Tremendous progress has been made in uncovering extracellular signals and the receptors that regulate neurite outgrowth and growth cone guidance (Tessier-Lavigne and Goodman, 1996). As reflected in the ability of cyclic nucleotide levels to switch guidance cues from attractive to repulsive, the rapid extension and retraction of processes associated with neurite growth requires careful coordination (Song and Poo, 1999). We wondered whether this task involved neuronal proteins containing multiple GEF domains.

Kalirin, a Dbl family member with multiple isoforms, is highly expressed in adult rat CNS (Alam et al., 1997). The longest Kalirin protein, Kalirin-12, contains a Sec14-like putative lipid binding domain, nine spectrin-like repeats, tandem $\mathrm{DH}$ and $\mathrm{PH}$

Received June 19, 2001; revised Aug. 1, 2001; accepted Aug. 7, 2001.

This work was supported by National Institutes of Health Grants DA-00266 and DK-32948. We thank Dr. L. Redmond for her help with neuronal transfections. We thank Drs. A. Ghosh, A. Kolodkin, and C. Hopf for their useful advice and for critically reading this manuscript.

Correspondence should be addressed to Betty A. Eipper, Department of Neuroscience, University of Connecticut Health Center, 263 Farmington Avenue, Farmington, CT 06030. E-mail: eipper@uchc.edu.

Copyright (C) 2001 Society for Neuroscience $0270-6474 / 01 / 218426-09 \$ 15.00 / 0$
GEF domain alone induces axonal over-elongation and abundant filopodial neurites, mediated by RhoA. Coordination of the actions of the individual GEF domains through their presence in Kalirin-9, with its Sec14p, spectrin, and Src homology domain 3 motifs, is essential for regulating neurite extension and neuronal morphology.

Key words: Rac1; RhoA; Trio; spectrin; cytoskeleton; growth cone

domains, an Src homology domain 3, a second tandem DHPH domain, a region of Ig-like repeats, and a serine-threonine kinase-like domain (see Fig. 1a). Kalirin-9 and Kalirin-12, the major isoforms expressed during embryonic development (Hansel et al., 2001), both contain two DH domains, whereas Kalirin-7, the major adult isoform, contains only one (Johnson et al., 2000; Penzes et al., 2000).

Studies in Drosophila and C. elegans indicate that a protein homologous to Kalirin-9 (dTrio and UNC-73, respectively) is an essential player in multiple axon guidance pathways (Steven et al., 1998; Awasaki et al., 2000; Bateman et al., 2000; Liebl et al., 2000; Newsome et al., 2000) (Fig. 1a). Mutational studies indicated an essential role for the first DHPH domain, with no clear role for the second DHPH domain (Steven et al., 1998; Bateman et al., 2000; Liebl et al., 2000; Newsome et al., 2000). In this study, we sought to understand the importance of having multiple DHPH domains included in a single protein capable of interacting simultaneously with several proteins and with membrane lipids.

\section{MATERIALS AND METHODS}

DNA and antibodies. Rac1-N17 and RhoA-N19 plasmids were gifts from Dr. Silvio Gutkind (National Institute of Dental Research, National Institutes of Health, Bethesda, MD), whereas glutathione $S$-transferase (GST)-RhoA, GST-Rac1, and GST-Cdc42 plasmids and constitutively active Rac1 (Rac1-Q61L) and RhoA (RhoA-Q63L) plasmids were gifts from Dr. Richard Cerione (Cornell University, Ithaca, NY). Rabbit polyclonal antisera against regions of Kalirin were affinity purified (Johnson et al., 2000; Penzes et al., 2000). Monoclonal antisera to neuronal-specific tubulin (Babco, Richmond, CA), Rac1 (Upstate Biotechnology, Lake Placid, NY), and RhoA (Santa Cruz Biotechnology, Santa Cruz, CA) were purchased. FITC-phalloidin was from Sigma (St. Louis, MO). pEGFP-N2 vector was from Clontech (Palo Alto, CA). C3 transferase expression vector was from Dr. Anirvan Ghosh (Johns Hopkins University, Baltimore, MD).

Cell cultures and transient transfections. NIH3T3 cells were cultured in DMEM-F-12 containing 10\% fetal bovine serum (HyClone, Logan, UT) and $10 \%$ NuSerum (Collaborative Research, Bedford, MA). For immunocytochemistry, cells grown on glass chamber slides for $3 \mathrm{~d}$ to $40-60 \%$ confluence were transfected with $1 \mu \mathrm{g}$ of plasmid DNA per 4 
$\mathrm{cm}^{2}$ of pEAK10.His-Myc-DHPH1, pEAK10.His-Myc-DHPH2, or pEAK10.His-Myc-Kal-9 and $4 \mu \mathrm{l}$ of Lipofectamine (Life Technologies, Gaithersburg, MD) in $1 \mathrm{ml}$ of complete serum-free medium for 5 $\mathrm{hr}$, after which they were washed and fed with growth medium. After $1 \mathrm{~d}$, medium was replaced with DMEM-F-12 for $16 \mathrm{hr}$. Cells fixed in $3.7 \%$ formaldehyde in PBS ( $50 \mathrm{~mm} \mathrm{NaPi}, \mathrm{pH} 7.5$, and $150 \mathrm{~mm} \mathrm{NaCl}$ ) for $30 \mathrm{~min}$ at room temperature were permeabilized and stained.

Neuronal cultures and transfections. Rat embryo cerebral cortex (embryonic day 18) digested with papain for $30 \mathrm{~min}$ at $37^{\circ} \mathrm{C}$ was dissociated by pipetting. Neurons were plated in chamber slides precoated with poly-L-lysine in MEM containing $2 \mathrm{~mm}$ glutamine and $5 \%$ heatinactivated horse serum (Life Technologies), penicillin, and streptomycin. After $2 \mathrm{~d}$ at $37^{\circ} \mathrm{C}$ in $5 \% \mathrm{CO}_{2}$, cultures were transfected using the calcium phosphate method for 15 min (Penzes et al., 2001). Cells in one chamber of a two-chamber slide were transfected with $1 \mu \mathrm{g}$ of pEGFP-N2 and $1 \mu \mathrm{g}$ of a pEAK10.His-Myc Kalirin-encoding vector. Vectors encoding Rho GTPases $(2-3 \mu \mathrm{g})$ were transfected along with pEGFP-N2 (1 $\mu \mathrm{g})$ and, where indicated, a Kalirin-encoding vector $(1 \mu \mathrm{g})$. After $2 \mathrm{~d}$ at $37^{\circ} \mathrm{C}$, neurons were fixed in $3.7 \%$ formaldehyde for $20 \mathrm{~min}$, permeabilized, and blocked in PBS containing 2\% normal goat serum and $0.1 \%$ Triton X-100 for $1 \mathrm{hr}$. Antibody was added in PBS with 2\% normal goat serum for $2 \mathrm{hr}$ at room temperature. Transfected myc-tagged Kalirin constructs were visualized by immunostaining with monoclonal or polyclonal myc antibodies. Morphological features were quantified with the help of an independent observer using the IPLab software (Scanalytics Inc., Fairfax, VA). Pictures of randomly selected fields were taken at low magnification, and the length of the longest process on each individual neuron in the field was measured after it was traced using the computer program; at least 30 neurons from two to three independent transfections were analyzed. In addition, transfected neurons were evaluated for the presence of growth cones on the cell soma and filopodial structures along neurites. A neuron was scored as positive if it had one or more growth cones and abundant filopodial structures.

Rac1 GTPase activation assays. Activation of Rac1 was measured with the Rac1/Cdc42 activation assay kit (Upstate Biotechnology). Briefly, cells that had been transfected $1 \mathrm{~d}$ earlier were serum-starved overnight, and cell lysates $\left(\sim 0.5 \mathrm{mg}\right.$ of protein) were incubated for $1 \mathrm{hr}$ at $4^{\circ} \mathrm{C}$ with $10 \mu \mathrm{l}$ of GST-PAK1-PBD resin per sample. Bound proteins were eluted and analyzed by SDS-PAGE and Western blotting with Rac1 and Cdc42 monoclonal antibodies.

DHPH binding to Rho GTPases. The GST fusion proteins of Rho GTPases purified from Escherichia coli were depleted of bound nucleotide by incubation in $10 \mathrm{~mm}$ EDTA. pEAK-rapid human embryonic kidney 293 (HEK-293) cells expressing myc-DHPH1 or myc DHPH2 were extracted in binding buffer $(20 \mathrm{~mm}$ Tris- $\mathrm{HCl}, \mathrm{pH} 7.5$, and $50 \mathrm{~mm}$ $\mathrm{NaCl}$ containing $1 \%$ Triton $\mathrm{X}-100$ ). For each binding reaction, $5 \mu \mathrm{g}$ of GST-GTPase bound to $25 \mu \mathrm{l}$ of glutathione-Sepharose beads was mixed with an aliquot of cell extract containing $1 \mathrm{mg}$ of protein for $1 \mathrm{hr}$ at $4{ }^{\circ} \mathrm{C}$. The beads were washed three times with binding buffer, and bound proteins eluted with Laemmli's buffer were analyzed by SDS-PAGE and Western blotting using the monoclonal myc antibody.

Tissue preparation and subcellular fractionation. Parietal cortex dissected from Sprague Dawley (Harlan Sprague Dawley, Indianapolis, IN) rat pups was sonicated in 10 vol of buffer A: $20 \mathrm{~mm}$ Tris $\mathrm{HCl}, \mathrm{pH} 7.5,1 \%$ SDS, $0.3 \mathrm{mg} / \mathrm{ml}$ PMSF, $50 \mu \mathrm{g} / \mathrm{ml}$ lima bean trypsin inhibitor, $2 \mu \mathrm{g} / \mathrm{ml}$ leupeptin, $16 \mu \mathrm{g} / \mathrm{ml}$ benzamidine, and $2 \mu \mathrm{g} / \mathrm{ml}$ pepstatin. Cultured primary neurons were extracted in the same buffer. Samples were prepared by addition of $2 \mathrm{vol}$ of $1 \times$ SDS-PAGE loading buffer and analyzed by SDS-PAGE and Western blotting.

\section{RESULTS}

\section{Expression of Kalirin isoforms with single and dual DHPH domains}

To determine when Kalirin isoforms with single or dual DHPH domains are expressed, we used a Kalirin antibody directed to the common spectrin-like domains to evaluate expression during postnatal development (Fig. $1^{1} b$ ). Forms with two DHPH domains, Kalirin-9 and Kalirin-12, are prevalent at postnatal day 2 (P2), a time when they could play a role in neural development. Expression of Kalirin-7, with a single DHPH domain, is not detectable until 2 weeks after birth, with increasing levels thereafter, consistent with its reported function in generation of spine-
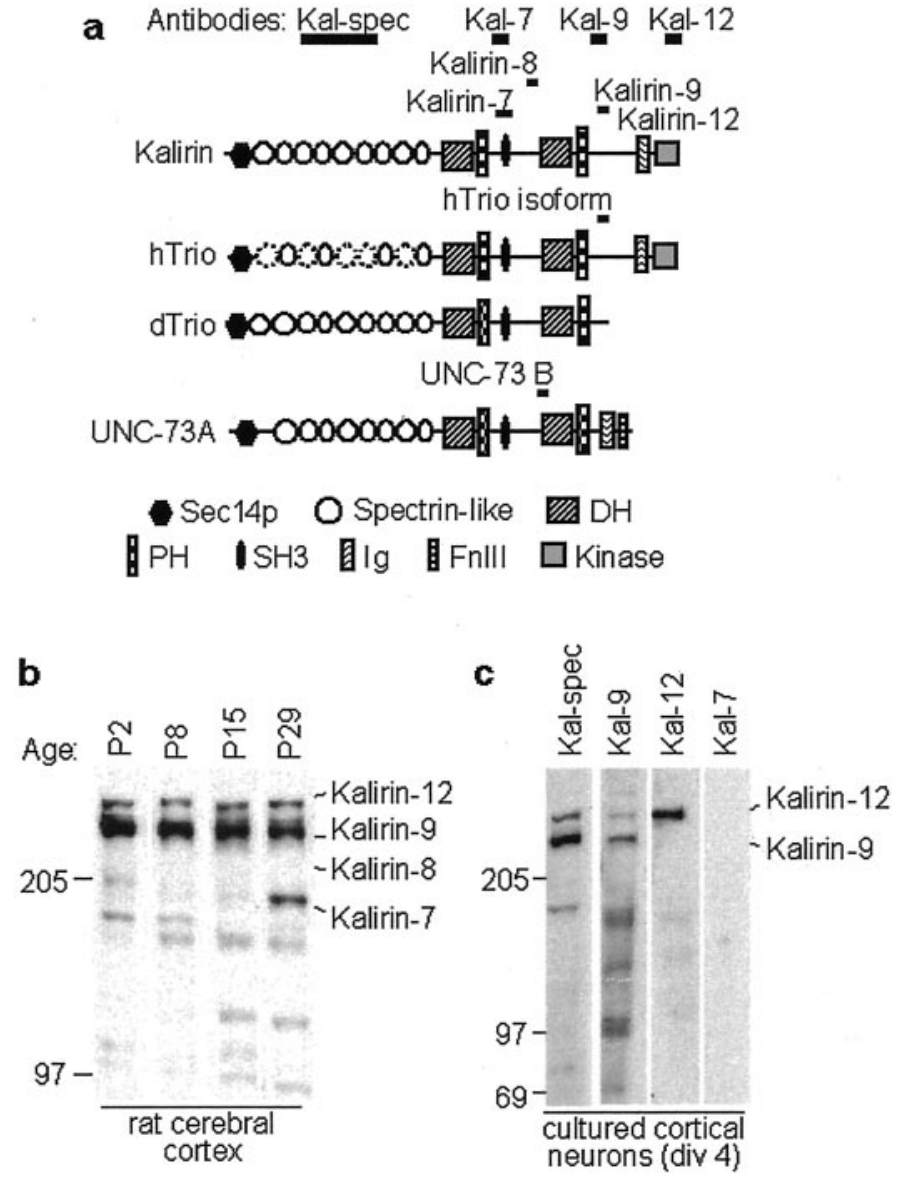

Figure 1. Expression of Kalirin. $a$, Kalirin isoforms and related proteins. Bars indicate antigens used to generate antibodies. $b$, Expression of Kalirin in rat cerebral cortex $(25 \mu \mathrm{g}$ of protein) during postnatal development, detected with the Kal-spec antibody (1:1000). $c$, Expression of Kalirin isoforms in 4-d-old cultures of dissociated cortical neurons ( $25 \mu \mathrm{g}$ of protein), detected with the indicated Kalirin antibodies. Kal-spec was used at 1:1000; Kalirin-9, Kalirin-12, and Kalirin-7 affinity-purified antibodies were used at 1:100. Kalirin-7 cannot be detected at this age. FnIII, Fibronectin III; div, days in vitro.

like structures in mature hippocampal neurons (Penzes et al., 2001). This experiment suggests that isoforms of Kalirin, with potentially different functions, are generated by developmentally regulated alternative splicing of a single gene product.

Because we wanted to use cultured neurons to study the function of Kalirin and its isolated DHPH domains, we evaluated extracts of 4-d-old dissociated cortical cultures using several Kalirin antibodies (Fig. 1c). The expression patterns of Kalirin isoforms in primary cortical cultures and $\mathrm{P} 2$ brain extracts are similar; forms of Kalirin with two DHPH domains predominate, and Kalirin-7 is not detectable (Fig. 1c).

\section{Kalirin is a dual-function GEF}

The first DHPH domain of Kalirin (DHPH1) is similar to the first DHPH domain of Trio (Debant et al., 1996) and UNC-73 (Steven et al., 1998), both of which are GEFs for Rac1. The second DHPH domain of Kalirin (DHPH2) is most similar to the second DHPH domain of Trio and UNC-73, both of which are GEFs for RhoA. To get an indication of the GEF activity of the DHPH domains of Kalirin, we tested the binding specificities of these domains for nucleotide-depleted Rho GTPases (Fig. 2a). We expressed myc-tagged DHPH1 or DHPH2 in pEAK-rapid 


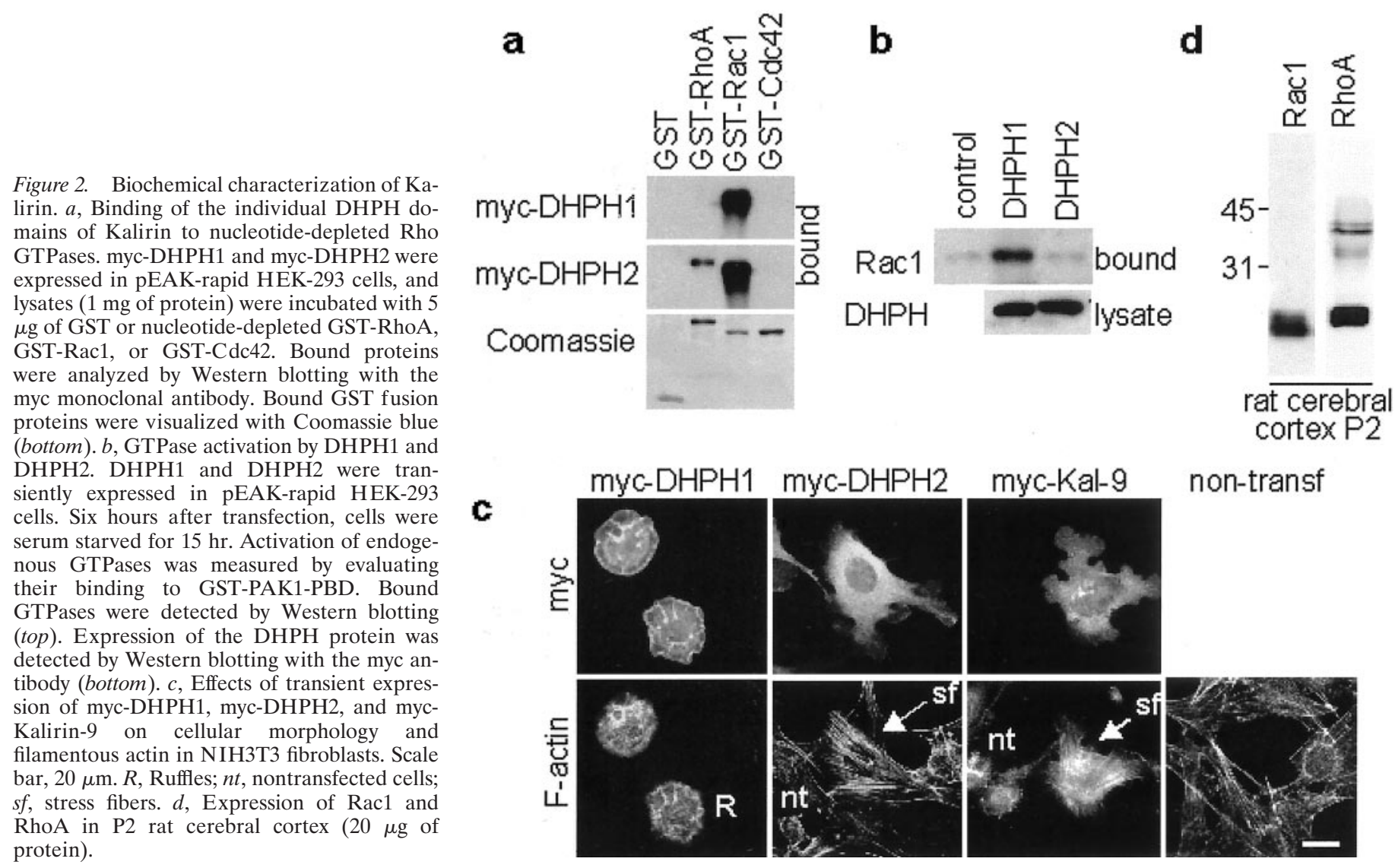

HEK-293 cells and performed binding experiments to nucleotide-depleted GST fusion proteins of RhoA, Rac1, and Cdc42. As determined previously (Alam et al., 1997), DHPH1 bound to nucleotide-depleted Rac1 but not to RhoA or Cdc42. This is consistent with its activity as a Rac1 GEF in vitro and in cells (Penzes et al., 2000). DHPH2 bound to nucleotide-depleted RhoA but surprisingly also bound to Rac1. DHPH2 did not bind to $\mathrm{Cdc} 42$.

Because both DHPH1 and DHPH2 bound nucleotidedepleted Rac1, we examined the activation of Rac1 and Cdc42 by DHPH1 and DHPH2. We expressed myc-tagged DHPH1 or DHPH2 in pEAK-rapid HEK-293 cells and measured the activation of endogenous Rac1 (Fig. 2b) and Cdc42 (data not shown) by affinity isolation of their activated forms. Expression of the DHPH1 domain resulted in activation of Rac1 but not $\mathrm{Cdc} 42$. On the contrary, expression of DHPH2 at the same level activated neither Rac1 nor Cdc42. As for DHPH2, other Dbl family members have been found to bind to Rho family members without catalyzing nucleotide exchange (Whitehead et al., 1997).

We used fibroblasts to compare the actions of the isolated DHPH domains of Kalirin with those of full-length Kalirin-9 (Fig. 2c). We transfected fibroblasts with myc-tagged Kalirin constructs. After serum starvation, we detected expression of the Kalirin proteins by immunostaining for the myc tag and examined the actin cytoskeleton by staining with FITC-phalloidin. As reported, DHPH1-transfected cells are disc shaped and display lamellipodia and ruffles, indicative of activated Rac1 (Hall, 1998; Penzes et al., 2000). In contrast, fibroblasts expressing DHPH2 exhibit abundant stress fibers, indicative of RhoA activation. Nontransfected cells do not display lamellipodia and have fewer stress fibers. Thus, the two DHPH domains of Kalirin interact with small GTPases in different ways to regulate the actin cytoskeleton.

Full-length Kalirin-9, which includes both DHPH domains, induced a phenotype distinct from that of either DHPH domain alone. The formation of both lamellipodia and stress fibers was increased when Kalirin-9 was transfected into fibroblasts. Cells expressing Kalirin-9 did not exhibit the massive ruffles seen in cells expressing DHPH1, suggesting that the DHPH1 domain may not be as active when part of the larger protein. This experiment suggests that both DHPH domains are activated to some extent in serum-starved fibroblasts.

To evaluate the expression of the potential Kalirin substrates in cortical cultures and to test the specificities of the commercial monoclonal antibodies for Rac1 and RhoA, we probed Western blots with monoclonal antibodies specific for Rac1 and RhoA (Fig. 2d). Both Rho proteins were readily detected, with both antibodies visualizing doublets of $\sim 21 \mathrm{kDa}$ proteins, showing that the antibodies are specific.

\section{Kalirin forms are compartmentalized in neurons}

To examine the distribution of endogenous Kalirin in cultured cortical neurons, we used form-specific Kalirin antisera and neuron-specific tubulin antiserum (Fig. 3a,b). We examined young ( $\mathrm{P} 0-\mathrm{P} 2)$ neurons and found that essentially all of the neurons expressed both Kalirin-9 and Kalirin-12. Whereas endogenous Kalirin-9 was abundant in processes, along with the cell soma (Fig. 3a), endogenous Kalirin-12 was exclusively localized to the cell soma (Fig. 3b). Staining of neurons with Kalirin-9 antibodies along with the dendritic marker microtubuleassociated protein 2 (MAP2) or the axonal marker tau showed 

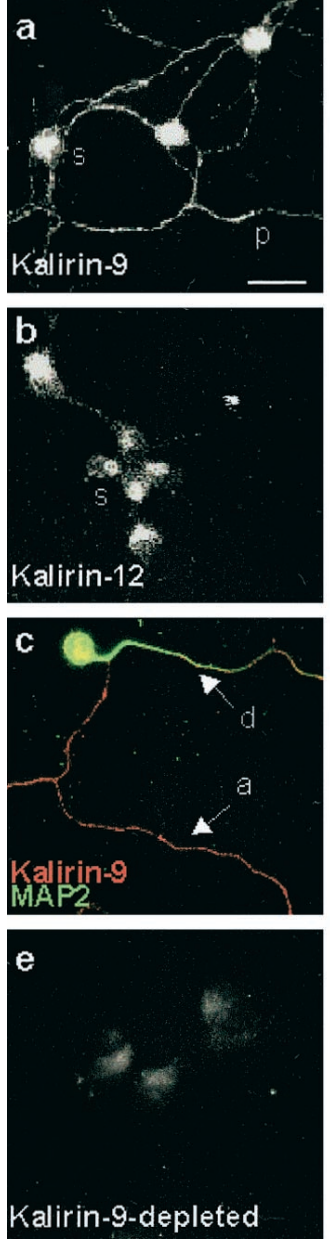
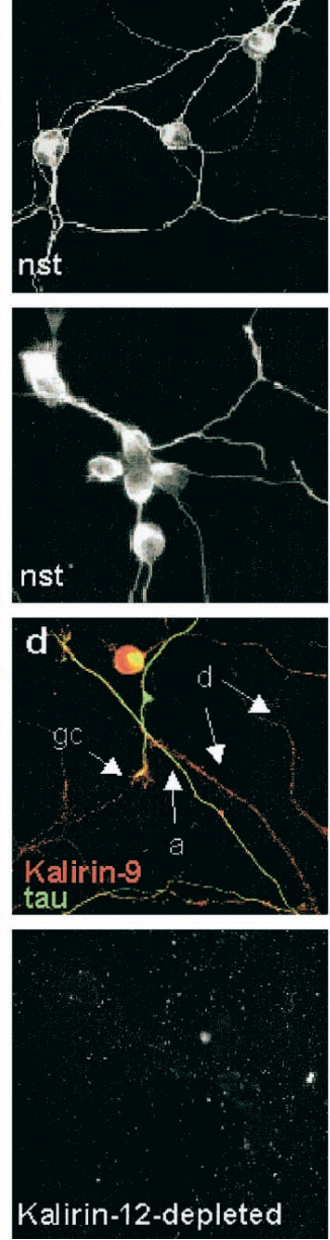
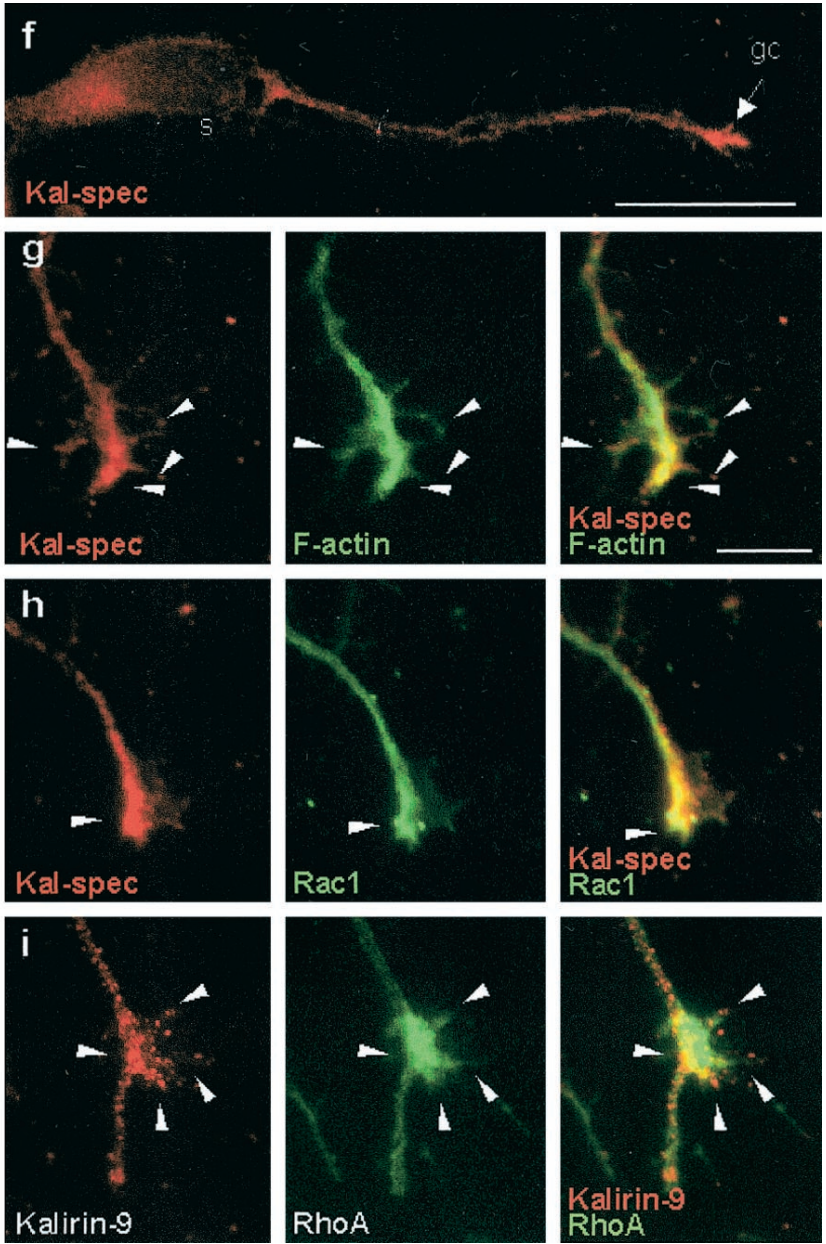

Figure 3. Compartmentalization of endogenous Kalirin. Cultured cortical neurons ( $2 \mathrm{~d}$ in vitro $)$ were stained simultaneously for neuron-specific tubulin $(n s t)(a, b)$ and Kalirin-9 $(a)$ or Kalirin-12 (b), MAP2 and Kalirin-9 (c), and tau and Kalirin-9 $(d)$. e, Immunostaining with Kalirin-9 and Kalirin-12 antibody solutions depleted by binding to antigen-affinity resins. $f$, Immunostaining of cultured neurons with Kal-spec antibody. Simultaneous immunostaining of cultured neurons for Kal-spec and F-actin $(g)$, Rac1 ( $h$ ), or RhoA ( $i)$. Scale bars: $a-f, 20 \mu \mathrm{m} ; g-i, 5 \mu \mathrm{m}$. $p$, Processes; $s$, soma; $a$, axons; $d$, dendrites; gc, growth cones; arrowheads, growth cone filopodia.

that Kalirin-9 was abundant in both dendrites and axons (Fig. $3 c, d)$. Depletion of both antisera by binding to affinity resins containing the corresponding antigenic peptide confirmed that the antibodies detected the desired epitopes (Fig. 3e). Because the two dual DHPH domain forms of Kalirin are localized differently, each may play a distinct role in regulating neuronal process growth.

Because the Kalirin-9 antibody showed several crossreactive bands on Western blots, we corroborated these observations by immunostaining cultures with the Kalirin-spectrin (Kal-spec) antibody (Fig. 3f). Kalirin was visualized in growth cones $(g c)$ and in the perinuclear region of the cell soma $(s)$. Kalirin was abundant in the growth cones (Fig. $3 g-i$, arrowheads), in which it colocalized with filamentous actin (F-actin) (Fig. $3 g$ ). Rac1 and RhoA, potential Kalirin substrates, are also localized to growth cones (Fig. 3h,i). Because Kalirin-12 is primarily localized to the cell soma (Fig. $3 b$ ) and Western blot analysis indicates that Kalirin-9 is the major isoform, growth cone staining is likely to represent Kalirin-9. Thus, Kalirin-9 is located where it could regulate actin assembly via available Rho protein substrates.

\section{Kalirin-9 and its individual DHPH domains have different effects on neuronal process length and morphology}

Based on the function of invertebrate proteins related to Kalirin and the striking subcellular localization of Kalirin in neurites and growth cones, we first compared the ability of Kalirin-9 and its individual DHPH domains to affect neurite extension. We expressed green fluorescent protein (GFP) (control) (Fig. 4a) and myc-tagged Kalirin-9 (Fig. 4b) in young cortical neurons (P0). We evaluated the effect of overexpressing myc-Kalirin- 9 by measuring the length of the longest process in the population of neurons visualized with the myc antibody. The longest processes in mycKalirin-9-expressing neurons were significantly longer than the longest processes of control neurons expressing GFP alone; GFP was visualized by autofluorescence (Fig. $4 c$ ).

To evaluate the contribution of the individual DHPH domains to the function of Kalirin-9, we overexpressed each DHPH domain in young cortical neurons (Fig. 4d,e). Expression of DHPH1 resulted in neurons with significantly shorter processes (Fig. $4 d, g$, top) than control neurons (Fig. 4c, top,$f$ ). In contrast, expression of DHPH2 (Fig. 4e,g, bottom) resulted in a larger number of 

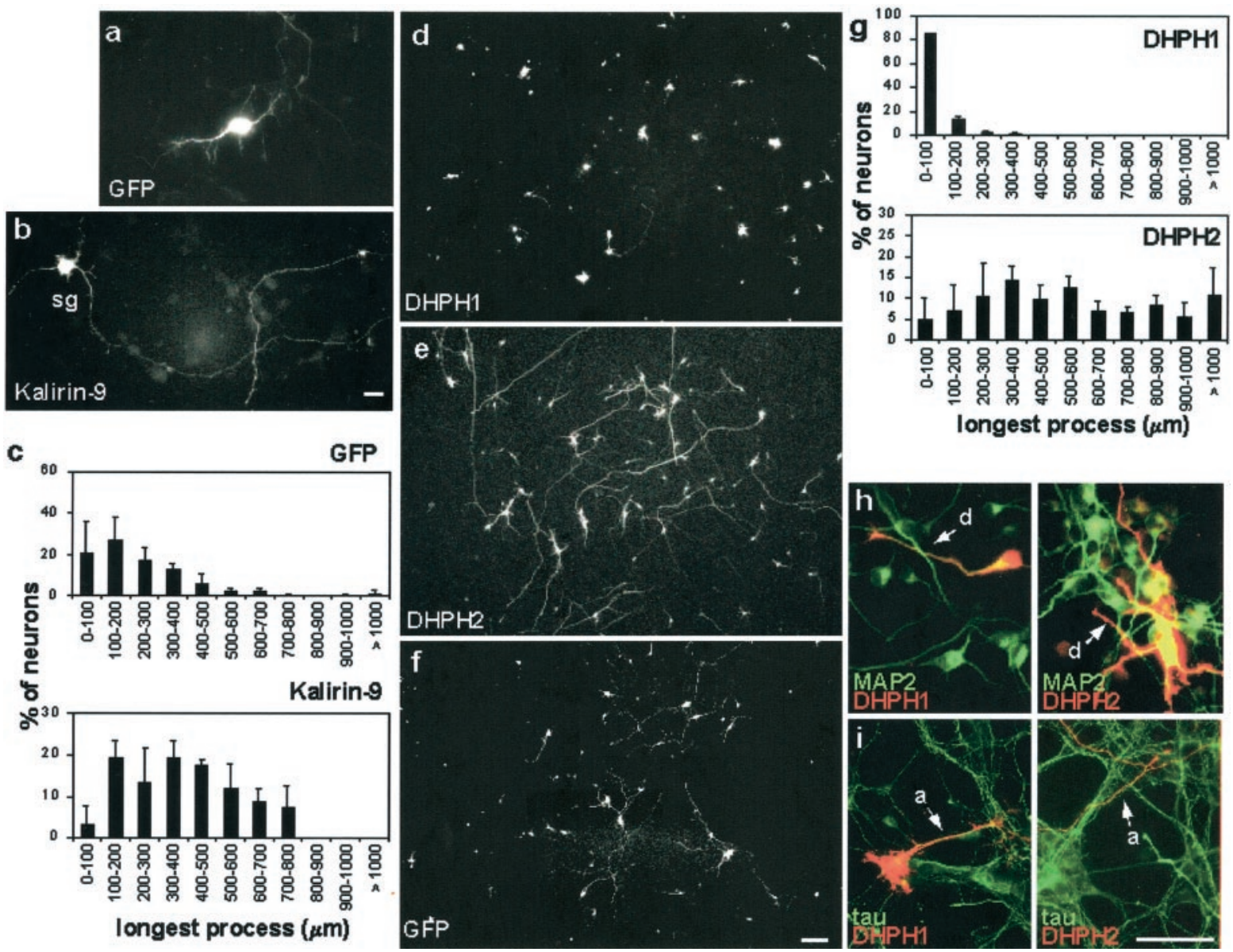

Figure 4. Overexpression of Kalirin-9 and its individual DHPH domains affects length of neurites. Cortical neurons $(2 \mathrm{~d}$ in vitro $)$ were transfected with GFP $(a)$ or myc-Kalirin-9 $(b)$. Scale bar, $10 \mu \mathrm{m}$. $c$, The length of the longest process in each Kalirin-9- or GFP-expressing neuron was determined. Low-magnification pictures of cortical cultures transfected with myc-DHPH1 $(d)$, myc-DHPH2 $(e)$, or GFP $(f)$. Scale bar, $100 \mu$ m. $g$, Histograms of lengths of the longest process of transfected neurons. For $c$ and $g$, error bars are SDs for three experiments, each containing 40-80 neurons. Cultured cortical neurons transfected with myc-DHPH1 $(h, i$, left $)$ or myc-DHPH2 $(h, i$, right $)$ were stained for myc and MAP2 $(h)$ or myc and tau $(i)$. Scale bar, $20 \mu$ m.

neurons with much longer processes than control neurons, Kalirin-9-transfected neurons, or neurons expressing DHPH1. For neurons transfected with myc-tagged DHPH1 or DHPH2, myc staining filled dendrites to the same extent as MAP2 staining and axons to the same extent as tau staining. Based on staining intensity, the expression levels of transfected DHPH1 and DHPH2 were similar.

To determine whether the processes affected by overexpression of the DHPH domains were axons or dendrites, we stained transfected neurons with the dendritic marker MAP2 (Fig. 4h, arrow $d$ ) or with the axonal marker tau (Fig. 4i, arrow a). Some of the longest processes of neurons transfected with DHPH1 stained with the MAP2 antibody, whereas others stained with the tau antibody (Fig. 4h,i), showing that expression of DHPH1 affected the length of both types of processes. In contrast, the longest processes of DHPH2-transfected neurons stained for tau but not for MAP2, indicating that they were axons (Fig. 4h,i). Thus, expression of the individual DHPH domains of Kalirin affected the growth of axons in opposite ways: DHPH1 expression resulted in a drastic reduction in length or disappearance of the axon, whereas DHPH2 expression induced an exaggerated extension of the axon.

Other morphological alterations were apparent after expression of Kalirin-9, DHPH1, and DHPH2 in cortical neurons.
Neurons overexpressing Kalirin-9 often displayed multiple lateral growth cones extending from neurites (Fig. 5a). A large fraction of neurons overexpressing Kalirin-9 had growth cones on their cell soma. An even larger fraction of neurons expressing DHPH1 had growth cones on their cell body (Fig. $5 b$, inset, $g c$; quantified in $e$ ); neurons expressing DHPH2 (Fig. $5 c$ ) or GFP (Fig. $5 d$ ) did not exhibit a similar morphology. DHPH1-expressing neurons, although they had been in culture for $4 \mathrm{~d}$, were reminiscent of the first stages of emergence of neurites.

Neurons expressing DHPH2 had many small aberrant filopodial neurites $(f n)$ and shorter and thinner than normal processes, densely covering the soma (Fig. $5 c$, inset; quantified in $f$ ), dendrites, and axon. Such structures were much less abundant on neurons expressing GFP, DHPH1, or Kalirin-9. Localization of endogenous Kalirin-9 to growth cones and small neuritic filopodia along processes places it at the sites of action of its overexpressed DHPH domains. These observations suggest that endogenous Kalirin-9 may generate these structures by selective activation of its DHPH1 and DHPH2 domains.

\section{DHPH1 and DHPH2 actions involve Rac1 and RhoA, respectively}

To determine whether the effects of DHPH1 and DHPH2 on neurons involve the small GTPases Rac1 and RhoA, we used 
a
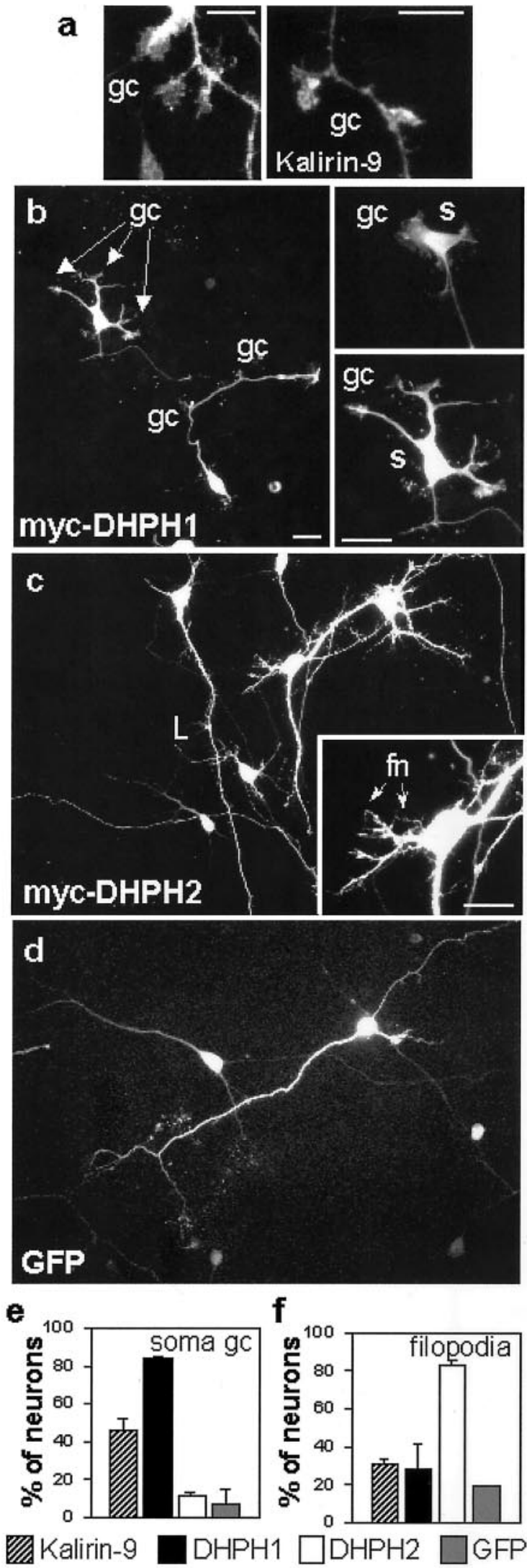

Figure 5. Expression of Kalirin-9 and its DHPH domains affects the morphology of cortical neurons. Morphological features induced by overexpression of Kalirin-9 (a), DHPH1 (b), DHPH2 $(c)$, and GFP $(d)$. Scale bars: $a, 10 \mu \mathrm{m} ; b-d, 20 \mu \mathrm{m}$. $g c$, Growth cones; $s$, soma; $f n$, aberrant filopodial neurites. $e$, Percentage of neurons with one or more growth dominant negative constructs of Rac1 and RhoA (Ridley and Hall, 1992; Nobes and Hall, 1995) (Fig. 6a). Coexpression of DHPH1 with the dominant negative mutant of Rac1 (Rac1$\mathrm{T} 17 \mathrm{~N})$ yielded neurons with significantly longer processes than those expressing DHPH1 alone (Figs. 4g, 6a, top panels). Coexpression of DHPH2 with dominant negative RhoA (RhoA-T19N) resulted in a reduction in the length of the longest process to a size similar to control GFP neurons (Figs. 4g, 6a, bottom panels). Coexpression of DHPH1 with RhoA-T19N or DHPH2 with Rac1-T17N failed to interfere with the effect of the DHPH domain (data not shown). Thus, the effects of DHPH1 on process growth involve an essential Rac1-mediated step, whereas those of DHPH2 involve an essential RhoA-mediated step.

Our results regarding the effects of Rac1 and RhoA on neurite outgrowth in cortical cultures were not those predicted based on some previous studies (van Leeuwen et al., 1997; Li et al., 2000) but are similar to studies on dorsal root ganglion neurons, in which Rac1 mediated growth cone collapse (Jin and Strittmatter, 1997). We verified our results by remapping the plasmids and testing multiple plasmid preparations. In addition, we overexpressed GFP along with dominant negative forms of Rac1 and RhoA in the cortical cultures. The length of longest process was indistinguishable after overexpression of dominant negative Rac1-T17N or RhoA-T19N alone (Fig. 6b). These data support our observation that DHPH1, acting through activation of Rac1, produces shorter neurites, whereas DHPH2, acting through activation of RhoA, produces longer neurites in primary cortical neurons.

To confirm that DHPH2 action involved activation of RhoA, we cotransfected neurons with vectors encoding myc-DHPH2 and C3 enzyme, a Clostridium botulinum ADP-ribosyltransferase that ADP-ribosylates Rho proteins and inactivates RhoA (Aktories et al., 1987). These neurons had overall morphologies very similar to that of controls (Fig. 6c); they lacked aberrant filopodia, suggesting that these structures were generated by a mechanism involving activation of RhoA. The longest processes of these neurons were significantly shorter than those of neurons expressing DHPH2 alone (Fig. $6 f$ ). This suggests that the actions of DHPH2 leading to process extension also require activation of RhoA. The fact that process lengths were shorter than control levels after expression of DHPH2 and C3 transferase and yet were unaffected by expression of dominant negative RhoA may reflect the ability of C3 transferase to inactivate additional Rho family members, such as RhoB and RhoC, that play may important roles in process outgrowth (Sekine et al., 1989).

To examine the behavior of the two DHPH domains in the context of full-length Kalirin-9, we cotransfected neurons with Kalirin-9 along with Rac1-T17N or RhoA-T19N, respectively. Overexpression of Kalirin-9 with Rac1-T17N resulted in neurons with cell bodies covered with small filopodial neurites (Fig. $6 d, f$ ), similar to those of neurons overexpressing DHPH2. The longest processes of these neurons were slightly longer than those of

\section{$\leftarrow$}

cones on soma. $f$, Percentage of neurons with abundant aberrant filopodial neurites extending from the soma. Bars represent averages of values for $\sim 50$ neurons, in three separate experiments; error bars are SDs. Cells were visualized by immunostaining with antisera for myc $(a-c)$ or by GFP autofluorescence $(d)$ cones on soma. $f$, Percentage of neurons with abundant aberrant filopodial neurites extending from the soma. Bars represent averages of values for $\sim 50$ neurons, in three separate experiments; error bars are SDs. Cells were visualized by immunostaining with antisera for myc $(a-c)$ or by GFP autofluorescence $(d)$. 

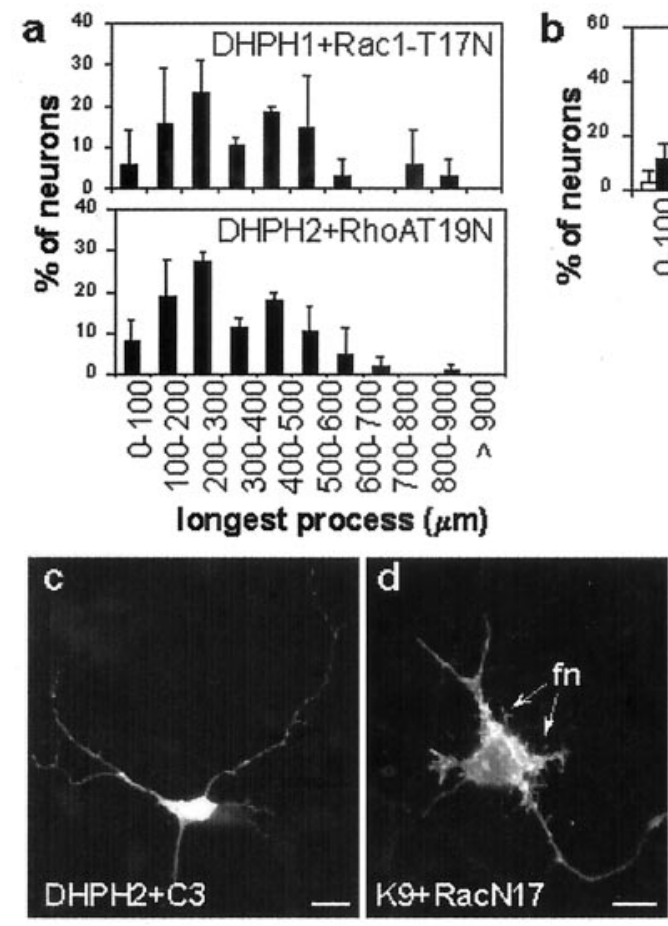

Figure 6. The actions of Kalirin-9 and its DHPH domains involve Rac1 and RhoA. $a$, Coexpression of dominant negative Rac1 and RhoA. Histogram of lengths of longest processes of neurons cotransfected with DHPH1 plus Rac1T17N or DHPH2 plus RhoA-T19N. Bars represent averages of $>30$ neurons in each experiment, and error bars are SDs for two experiments. $b$, Histogram of lengths of longest processes of neurons expressing dominant negative Rac1N17 and RhoA-N19 in the absence of exogenous Kalirin. Morphology of neurons transfected with DHPH2 plus C3 transferase $(c)$, Kalirin-9 plus Rac1-T17N $(d)$, or Kalirin-9 plus RhoA-T19N $(e)$. Histograms of lengths of longest processes of neurons cotransfected with DHPH2 plus C3 transferase $(f)$, Kalirin-9 plus Rac1-T17N ( $g$, top), or Kalirin-9 plus RhoA-T19N ( $g$, bottom). Bars represent averages of $>30$ neurons in each experiment, and error bars are SDs for two experiments.

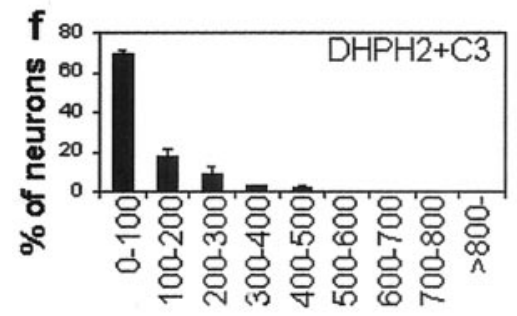

longest process (pm)
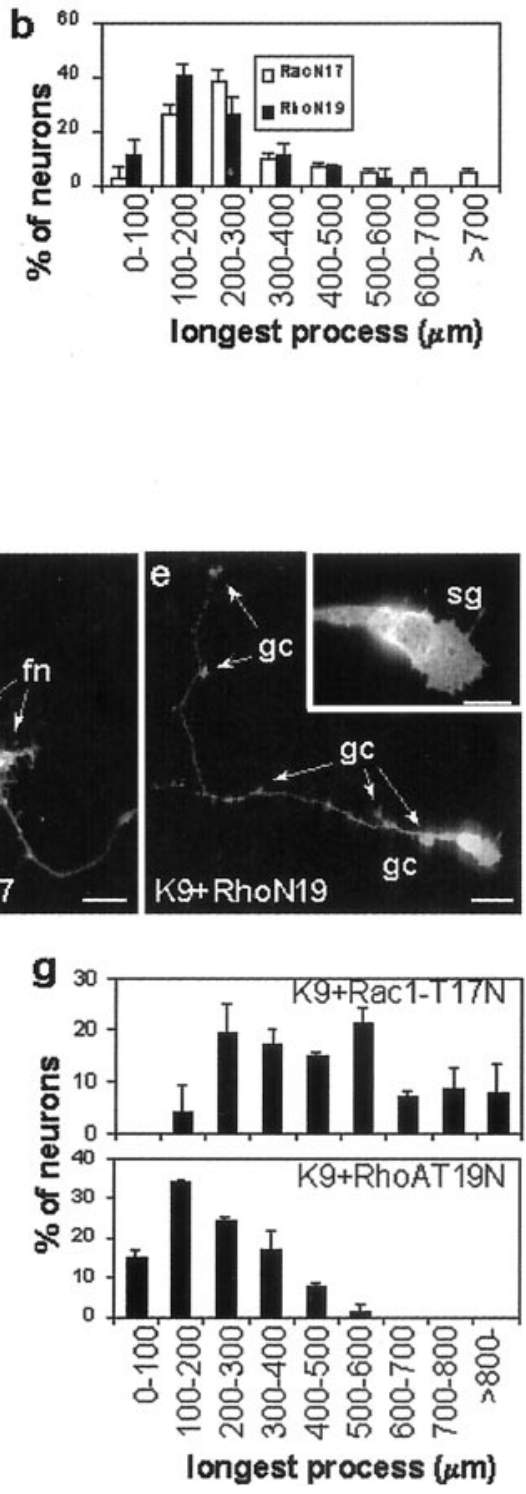

neurons overexpressing Kalirin-9 alone (Figs. 4c, 6g, top). Thus, the effects of Kalirin-9 on cortical neurons are mediated in part through activation of Rac1.

A considerable fraction of the neurons overexpressing Kalirin-9 and RhoA-T19N displayed massive lamellipodia on their soma and lateral growth cones along their neurites, reminiscent of the phenotype of neurons overexpressing DHPH1 (Fig. $6 e)$. Overexpression of Kalirin-9 with RhoA-T19N resulted in neurons with slightly shorter processes than those of neurons expressing Kalirin-9 alone (Fig. $6 g$, bottom). Thus, the effects of Kalirin- 9 are mediated in part through activation of RhoA. These results are consistent with the behavior of Kalirin-9 and its separate DHPH domains when transfected into NIH3T3 fibroblasts (Fig. 2c). Dominant negative Rac and Rho exerted a greater effect on neurons overexpressing Kalirin-9 than on control neurons (Fig. $6 b, g$ ); this may reflect the more controlled activation of endogenous Kalirin or the involvement of additional factors. Together, these experiments demonstrate that both DHPH domains are active in the context of the full-length protein. Protein localization or controlled activation of individual DHPH domains within the context of the full-length protein may account for the distinct effects of Kalirin-9.

\section{DISCUSSION}

An important role for dual DHPH isoforms of Kalirin in neurite growth in cortical neurons is supported by several lines of evidence. In the neonatal rat cortex, expression of Kalirin-9 and Kalirin-12 predominates, with little expression of Kalirin-7. Kalirin-9 is enriched in processes and growth cones of cortical neurons, with a striking localization to zones of intense growth. The dramatic effects of Kalirin-9 and its isolated DHPH1 and DHPH2 domains on neurite length and neuronal morphology implicate this protein in neuritogenesis, a process that takes place at the sites of localization of endogenous Kalirin-9. The homology of Kalirin-9 with Drosophila Trio and C. elegans UNC-73A, both involved in axon growth and guidance, implicates Kalirin in similar processes.

Whereas invertebrates have a single Kalirin/Trio gene, mammals have two, as revealed by database searches. In both Drosophila and C. elegans, Trio plays essential roles both within and outside the nervous system (Awasaki et al., 2000; Bateman et al., 2000; Liebl et al., 2000; Newsome et al., 2000). In the fly nervous system, dTrio may be involved in signaling from multiple extracellular cues (Awasaki et al., 2000; Bateman et al., 2000; Newsome 
et al., 2000). Whereas Trio null mice exhibit gross defects in skeletal muscle morphogenesis and few survive to birth (O'Brien et al., 2000), neuronal defects in Trio null mice are restricted to the dentate gyrus and mitral cell layer of the olfactory bulb. Thus, many of the neuronal functions ascribed to invertebrate Trio may be performed in mammals by Kalirin (O'Brien et al., 2000).

In Drosophila and C. elegans, expression of the dual DHPH proteins dTrio and Unc-73 is essential for axon growth and guidance (Steven et al., 1998; Awasaki et al., 2000; Bateman et al., 2000; Liebl et al., 2000; Newsome et al., 2000). In the systems examined, inactivation of the DHPH1 domain gave the same phenotype as the full knock-out, leading to the conclusion that the DHPH2 domains of dTrio and Unc-73 were not essential (Steven et al., 1998; Bateman et al., 2000; Liebl et al., 2000; Newsome et al., 2000). In the absence of the $\mathrm{NH}_{2}$-terminal spectrin domains of dTrio, a constitutively active phenotype was observed for the DHPH1 domain, and the phenotype was unaltered by including the DHPH2 domain as well (Newsome et al., 2000). Despite the fact that it exhibited no exchange factor activity when tested with six Rho GTPases, expression of the second DHPH domain of dTrio induced stress fiber formation in rat embryo fibroblasts, a phenotype generally indicative of RhoA activation (Newsome et al., 2000). Similarly, the DHPH2 domain of Kalirin induced stress fiber formation in fibroblasts. Unlike dTrio and Unc-73, both DHPH domains of Kalirin play a role in neonatal cortical neurons; Kalirin DHPH1 reduces axonal length, whereas Kalirin DHPH2 induces dramatic axonal overelongation and aberrant filopodial neurites.

Neurons encounter spatial issues not faced by other cells. One way they could address these problems is by using dual-function GEFs such as Kalirin- 9 and Kalirin-12 to coordinate the actions of individual GEF activities. The actions of full-length Kalirin-9 are not simply the sum of the actions of its individual DHPH domains. The presence of two distinct GEF activities in a single protein, such as Kalirin-9, allows coordinated regulation of the morphological changes induced; this may be especially important for neurite extension versus retraction. In the context of the full-length Kalirin protein, both DHPH domains are active in neonatal cortical neurons. Neither domain appears to be maximally activated under control conditions, making it possible for each domain to be activated in a regulated manner. Extracellular attractive or repulsive cues may selectively activate one DHPH domain or the other, and Kalirin-9 may have its separate DHPH domains activated like a switch, to quickly respond to the growth needs of the neuron, by virtue of its localization to active zones of growth.

The actions of DHPH1 and DHPH2 in neonatal cortical neurons are different, more dramatic and more complex than those reported for Rac1 and RhoA alone. These differences may reflect localization of the DHPH proteins or their ability to interact with multiple Rho proteins. The ability of DHPH domains to bind Rho proteins without activating them can clearly play a role in the differences observed. Diverse effects of Rho GTPases on neuronal morphology have been reported previously (Luo et al., 1994; Jin and Strittmatter, 1997; Li et al., 2000). For example, activated Rac1 blocked axon growth in both Drosophila and mice (Luo et al., 1994, 1997), inhibited axon formation in Xenopus (Ruchhoeft et al., 1999), and caused growth cone collapse in embryonic chick (DRG) neurons (Jin and Strittmatter, 1997). In other cases, activated Rac1 induced growth of neurites (Kuhn et al., 1999), increased the number of primary and basal dendrites (Threadgill et al., 1997), and increased dendritic branching and dynamics ( $\mathrm{Li}$ et al., 2000). Although activated RhoA stimulated neurite growth in chick embryonic motor neurons (Kuhn et al., 1999) and increased the number of primary and basal dendrites in rat cortical neurons (Threadgill et al., 1997), it mediated neurite retraction, caused growth cone collapse, and inhibited neurite extension in chick embryo ciliary neurons (Yamashita et al., 1999).

The effect of any DHPH domain will depend on the complement of Rho proteins present in any given neuron at a particular time. In addition, the action of a particular Rho family member may vary with cell type and developmental stage. For example, $C$. elegans UNC-73B and Drosophila Still life (SIF) both activate Rac1, yet UNC-73 stimulates axon growth and SIF inhibits it (Sone et al., 1997). Tiam1-induced activation of Rac antagonizes Rho signaling, with a balance determining neuronal morphology (van Leeuwen et al., 1997). GEF signaling clearly cannot be reduced to simple bulk activation of Rho GTPases.

\section{REFERENCES}

Aktories K, Weller U, Chhatwal GS (1987) Clostridium botulinum type $\mathrm{C}$ produces a novel ADP-ribosyltransferase distinct from botulinum C2 toxin. FEBS Lett 212:109-113.

Alam MR, Johnson RC, Darlington DN, Hand TA, Mains RE, Eipper BA (1997) Kalirin, a cytosolic protein with spectrin-like and GDP/ GTP exchange factor-like domains that interacts with peptidylglycine alpha-amidating monooxygenase, an integral membrane peptideprocessing enzyme. J Biol Chem 272:12667-12675.

Awasaki T, Saitoh M, Sone M, Suzuki E, Sakai R, Ito K, Hama C (2000) The Drosophila Trio plays an essential role in patterning of axons by regulating their directional extension. Neuron 26:119-131.

Bateman J, Shu H, van Vactor D (2000) The guanine nucleotide exchange factor Trio mediates axonal development in the Drosophila embryo. Neuron 26:93-106.

Debant A, Serra-Pages C, Seipel K, OBrien S, Tang M, Park S-H, Streuli M (1996) The multidomain protein Trio binds the LAR transmembranetyrosine phosphatase, contains a protein kinase domain, and has separate rac-specific and rho-specific guanine nucleotide exchange factor domains. Proc Natl Acad Sci USA 93:5466-5471.

Hall A (1998) Rho GTPases and the actin cytoskeleton. Science 279:509-514.

Hansel DE, Quiñones ME, Ronnett GV, Eipper BA (2001) Kalirin, a GDP/GTP exchange factor of the Dbl family, is localized to embryonic nerve, muscle, and endocrine tissue during embryonic rat development. J Histochem Cytochem 49:833-844.

Jin Z, Strittmatter SM (1997) Rac1 mediates collapsin-1-induced growth cone collapse. J Neurosci 17:6256-6263.

Johnson RC, Penzes P, Eipper BA, Mains RE (2000) Isoforms of kalirin, a neuronal dbl family member, generated through use of different $5^{\prime}$ and $3^{\prime}$-ends along with an internal translational initiation site. J Biol Chem 275:19324-19333.

Kuhn TB, Brown MD, Wilcox CL, Raper JA, Bamburg JR (1999) Myelin and collapsin-1 induce motor neuron growth cone collapse through different pathways: inhibition of collapse by opposing mutants of rac1. J Neurosci 19:1965-1975.

Li Z, Van Aelst L, Cline HT (2000) Rho GTPases regulate distinct aspects of dendritic arbor growth in Xenopus central neurons in vivo. Nat Neurosci 3:217-225.

Liebl EC, Forsthoefel DJ, Franco LS, Sample SH, Hess JE, Cowger JA, Chandler MP, Shupert AM, Seeger MA (2000) Dosage-sensitive, reciprocal genetic interactions between the Abl tyrosine kinase and the putative GEF Trio reveal Trio's role in axon pathfinding. Neuron 26:107-118.

Luo L, Liao YJ, Jan LY, Jan YN (1994) Distinct morphogenetic functions of similar small GTPases: Drosophila Drac1 is involved in axonal outgrowth and myoblast fusion. Genes Dev 8:1787-1803.

Luo L, Jan LY, Jan YN (1997) Rho family GTP-binding proteins in growth cone signalling. Curr Opin Neurobiol 7:81-86.

Newsome TP, Schmidt S, Dietzl G, Keleman K, Åsling B, Debant A, Dickson BJ (2000) Trio combines with dock to regulate Pak activity during photoreceptor axon pathfinding in Drosophila. Cell 101:283-294.

Nobes CD, Hall A (1995) Rho, Rac, and Cdc42 GTPases regulate the assembly of multimolecular focal complexes associated with actin stress fibers, lamellipodia, and filopodia. Cell 81:53-62.

O'Brien SP, Seipel K, Medley QG, Bronson R, Segal R, Streuli M (2000) Skeletal muscle deformity and neuronal disorder in Trio exchange factor-deficient mouse embryos. Proc Natl Acad Sci USA 97:12074-12078.

Penzes P, Johnson RC, Alam MR, Kambampati V, Mains RE, Eipper BA 
(2000) An isoform of Kalirin, a brain-specific GDP/GTP exchange factor, is enriched in the postsynaptic density fraction. J Biol Chem 275:6395-6403.

Penzes P, Johnson RC, Sattler R, Zhang X, Huganir RL, Kambampati V, Mains RE, Eipper BA (2001) The neuronal Rho-GEF Kalirin-7 interacts with PDZ domain-containing proteins and regulates spine morphogenesis. Neuron 2001 29:229-242.

Ridley AJ, Hall A (1992) The small GTP-binding protein regulates the assembly of focal adhesions and actin stress fibers in response to growth factors. Cell 70:389-399.

Ruchhoeft ML, Ohnuma S, McNeill L, Holt CE, Harris WA (1999) The neuronal architecture of Xenopus retinal ganglion cells is sculpted by rho-family GTPases in vivo. J Neurosci 19:8454-8463.

Sekine A, Fujiwara M, Narumiya S (1989) Asparagine residue in the rho gene product is the modification site for botulinum ADPribosyltransferase. J Biol Chem 264:8602-8605.

Sone M, Hoshino M, Suzuki E, Kuroda S, Kaibuchi K, Nakagoshi H, Saigo K, Nabeshima Y-I, Hama C (1997) Still life, a protein in synaptic terminals of Drosophila homologous to GDP-GTP exchangers. Science 275:543-547.
Song H-J, Poo M-M (1999) Signal transduction underlying growth cone guidance by diffusible factors. Curr Opin Neurobiol 9:355-363.

Steven R, Kubiseski TJ, Zheng H, Kulkarni S, Mancillas J, Morales AR, Hogue CWV, Pawson T, Culotti J (1998) UNC-73 activates the Rac GTPase and is required for cell and growth cone migrations in $C$. elegans. Cell 92:785-795.

Tessier-Lavigne M, Goodman CS (1996) The molecular biology of axon guidance. Science 274:1123-1133.

Threadgill R, Bobb K, Ghosh A (1997) Regulation of dendritic growth and remodeling by Rho, Rac, and Cdc42. Neuron 19:625-634.

van Leeuwen FN, Kain HET, van der Kammen RA, Michiels F, Kranenburg OW, Collard JG (1997) The guanine nucleotide exchange factor Tiam1 affects neuronal morphology; opposing roles for the small GTPases Rac and Rho. J Cell Biol 139:797-807.

Whitehead IP, Campbell S, Rossman KL, Der CJ (1997) Dbl family proteins. Biochim Biophys Acta 1332:F1-F23.

Yamashita T, Tucker KL, Barde YA (1999) Neurotrophin binding to the p75 receptor modulates Rho activity and axonal outgrowth. Neuron 24:585-593. 